\section{Conhecimento, atitudes e práticas sobre tuberculose em prisões e no serviço público de saúde}

\section{Knowledge, attitudes and practices on tuberculosis in prisons and public health services}

Sérgio Ferreira Júnior'

\section{Helenice Bosco de Oliveira"}

Letícia Marin-Léon"

' Secretaria Municipal de Saúde da Prefeitura de Hortolândia, São Paulo, Brasil.

" Departamento de Medicina Preventiva e Social da Universidade Estadual de Campinas, São Paulo, Brasil.

\section{Resumo}

São poucos os estudos sobre o conhecimento da tuberculose (TB) entre os detentos, trabalhadores do sistema penitenciário e da rede pública de saúde (RPS), e aqueles realizados com outras populações apontam a falta do conhecimento sobre a doença como uma das principais barreiras para a percepção dos sintomas, diagnóstico precoce, adesão ao tratamento e cura. Objetivo: Analisar o conhecimento, atitudes e práticas sobre a TB em uma unidade prisional e na rede pública de saúde (RPS). Metodologia: Foi realizado estudo transversal com aplicação do questionário KAP (knowledge, atittudes and practices) na coleta de dados. Participaram 141 detentos, 115 funcionários do presídio e 158 da RPS. O programa Epi-Info versão 6.04 foi utilizado para comparação de proporções com significância estatística para $p<0,05$. Resultados: Foram observados conceitos equivocados sobre a doença entre os três grupos pesquisados. $\mathrm{Na}$ RPS foram detectados erros básicos sobre o conhecimento da TB, concluindo-se que há falhas nos treinamentos. O KAP mostrou-se eficaz na coleta de dados gerais sobre conhecimento, porém foi limitado e frágil nas informações sobre práticas e atitudes, não sendo aconselhável a sua utilização como instrumento único na coleta de dados sobre conhecimento, práticas e atitudes em TB. É sugerida sua utilização periódica como auxiliar nas atividades educativas e, considerando a elevada prevalência de TB entre detentos, aponta-se para a necessidade do envolvimento das Secretarias de Saúde na supervisão destas atividades educativas no sistema prisional

Palavras-chave: Tuberculose. Prisões. KAP. Conhecimento. Atitudes. Práticas. Serviço Público de Saúde. 


\section{Abstract}

There are few studies about tuberculosis (TB) knowledge among prisoners, penitentiary system workers and public health network (PRN). Those carried with other populations, point out that the lack of knowledge about the illness is one of the main barriers to the perception of the symptoms, early diagnosis, treatment adherence and cure. Objective: To analyze the knowledge, attitudes and practices about TB in a prison and in public health services (PHS). Methods: A cross sectional study was carried out and KAP (knowledge, attitudes and practices) questionnaire was applied to 141 prisoners, 115 prison's employees and 158 PHS workers. Epi-Info version 6.04 was used for comparison of proportions with statistic significance at $\mathrm{p}<0.05$. Results: Mistaken concepts on TB were observed among the three searched groups. PHS also showed basic errors on TB knowledge thus pointing out imperfections on training. Conclusion: KAP revealed efficient for data collection of general knowledge items but was limited on practices and attitudes and so its use as the only tool for data collection about knowledge, attitudes and practices on TB is not advisable. It is suggested its regular use to aid educational activities and considering the high prevalence of TB among prisoners, it is noted the need to involve the Departments of Health in the supervision of educational activities in the prison system.

Keywords: Tuberculosis. Prisons. KAP. Knowledge. Attitudes. Practices. Public Health Service.

\section{Introdução}

As populações confinadas, em especial a população privada de liberdade, representam um sério problema para o controle de doenças infectocontagiosas como a tuberculose (TB) e a Aids. ${ }^{1}$ Mesmo nas prisões, estes indivíduos não estão totalmente isolados pelos muros que as cercam; o vínculo com o mundo exterior se dá por meio do contato com suas visitas e com os funcionários do sistema prisional. Podem também se relacionar com a comunidade no cumprimento da pena em regime semiaberto, nos indultos e nas fugas ou ainda no retorno à liberdade após o cumprimento da pena. Os funcionários por sua vez, mantêm contato com seus familiares e a comunidade. ${ }^{2}$ Esta condição significa um grande risco de contaminação de mão dupla, ou seja, uma epidemia de TB não controlada no interior do presídio pode representar um grave risco para estes contatos e para sociedade como um todo. Por outro lado, uma TB contraída na comunidade pode desencadear uma epidemia na população penitenciária. ${ }^{3}$

Além disto, as regras próprias do ambiente prisional exercem influência na relação do doente com a sua própria doença. A TB neste contexto pode afetar o convívio social do detento, ${ }^{4}$ contribuindo para a subvalorização dos sintomas da doença.

Na população geral e na prisional, a TB é assunto que ainda causa desconforto nos dias atuais. Está associada à pobreza, isolamento, exclusão social, comportamento desregrado e amoral, e também à degenerescência social. Estes valores estão fortemente presentes no estigma das prisões. ${ }^{5-8}$

Atualmente, o tratamento é realizado predominantemente de forma ambulatorial expondo os trabalhadores da saúde ao contágio. Assim, o conhecimento destes indivíduos sobre a TB pode ser fundamental em relação à sua vulnerabilidade. ${ }^{9}$

São poucos os estudos sobre o conhecimento da TB entre os detentos, trabalhadores do sistema penitenciário e da rede pública de saúde (RPS), e os realizados com outras populações apontam a falta do conhecimento sobre a doença como uma 
das principais barreiras para a percepção dos sintomas, diagnóstico precoce, adesão ao tratamento e cura. ${ }^{10,11}$

O questionário KAP (Knowledge, Attitudes and Practices) ${ }^{12}$ tem sido utilizado para a coleta de dados sobre o conhecimento, atitudes e práticas em diversos problemas de saúde ou doenças, ${ }^{13}$ sobre o que é conhecido, acreditado e feito em relação a um tema específico. Este instrumento teve sua origem na década de 1950 e foi desenhado para estimar a resistência entre as diferentes populações em relação à idéia do planejamento familiar. ${ }^{13}$

A pesquisa KAP-TB pode ser elaborada, especificamente, para reunir informações sobre temas relacionados à TB e também incluir questões sobre as práticas de saúde em geral e as crenças em relação à doença, identificar os conhecimentos, padrões de lacunas, crenças culturais ou comportamentais que facilitam a compreensão e ação, bem como detectar problemas, barreiras ou obstáculos criados nos esforços de controle da TB. ${ }^{14}$ Os dados podem ser analisados quanti ou qualitativamente, dependendo dos objetivos do estudo. ${ }^{12}$

$\mathrm{O}$ uso deste instrumento estimulou a mobilização social e as pesquisas que envolvem o conhecimento, práticas e atitudes da população em relação à TB. Alguns destes estudos foram conduzidos pelos programas de controle de vários países onde há maior incidência da doença, com o objetivo de apoiar intervenções para mudança comportamental. ${ }^{12}$ Um papel cada vez mais importante e comum para as pesquisas KAP vem sendo o de fornecer dados essenciais que mostrem o impacto das atividades de advocacia, comunicação e mobilização social (ACMS).

Partindo do pressuposto de que os funcionários da RPS e dos presídios, pelo acesso aos treinamentos, teriam conhecimento adequado sobre a $\mathrm{TB}$, foi proposto este estudo cujo objetivo foi comparar o conhecimento, atitudes e práticas sobre a $\mathrm{TB}$ entre detentos e funcionários do complexo penitenciário e destes com os funcionários da rede pública de saúde (RPS).

\section{Metodologia}

Foi realizado estudo transversal com população composta por detentos e funcionários da unidade prisional Penitenciária P-III, de regime fechado, e funcionários da RPS do município de Hortolândia-SP. A Penitenciária P-III foi escolhida porque não havia participado de nenhum projeto de intervenção educativo para TB.

Não foi possível obter amostra estatística entre os detentos devido às condições da prisão e aos impositivos de segurança. Os detentos foram selecionados aleatoriamente, de acordo com a rotina e critérios da instituição penitenciária para translado até o parlatório. Entre os funcionários da unidade prisional e da RPS a participação foi por interesse.

Foi aplicado o roteiro $\mathrm{KAP}^{12}$ adaptado como um questionário semiestruturado, composto por questões fechadas e organizado em quatro blocos que abordaram a situação sociodemográfica e os seguintes aspectos relacionados à TB: antecedentes da doença, conhecimento, comportamento diante da possibilidade de contrair a TB e atitudes em relação aos doentes com TB. O questionário adaptado foi pré-testado na unidade Penitenciária PI do complexo prisional.

Entre detentos e funcionários do presídio, o instrumento foi aplicado individualmente por entrevistador. Na RPS foi autoaplicado e distribuído a todos os servidores lotados nas unidades de saúde selecionadas.

Os dados foram digitados no programa Epi-Info, versão 6.04, e realizadas as análises das frequências das variáveis, considerando como variável independente a condição prisional/funcional (detentos e funcionários do presídio/RPS). Para a comparação das categorias das variáveis dependentes segundo a variável independente foi utilizado o teste do qui-quadrado, com significância estatística para $\mathrm{p}<0,05$. Algumas das questões permitiram mais de uma resposta. As comparações realizadas foram entre detentos e funcionários do presídio e entre estes e os da RPS. 
Questões éticas: A pesquisa foi previamente autorizada pela Secretaria de Administração Penitenciária do Estado de São Paulo, Secretaria Municipal de Saúde de Hortolândia e aprovada pelo Comitê de Ética da Universidade Estadual de Campinas com parecer $N^{\circ}$ 942/2009 da Comissão de Ética em Pesquisa do Conselho Nacional de Saúde, conforme a resolução 196/96. Todos os participantes assinaram o Termo de Consentimento Livre e Esclarecido e foi garantido o sigilo das informações.

Não houve conflito de interesse na realização do estudo.

\section{Resultados}

Na unidade prisional pesquisada estavam lotados 233 servidores e, destes, 88 afastados por motivos médicos, transferências temporárias, férias ou licença prêmio, sendo entrevistados 115 do total ativo de 145 (79,3\%). O número de detentos informado pela administração foi de 1.153 homens, sendo entrevistados 141 (12,2\%). Por motivos ligados à segurança, a coleta de dados dos detentos precisou ser interrompida. Segundo o departamento de recursos humanos, o número de funcionários na RPS era de 1.216, com 208 afastados, sendo entrevistados 158 entre os $508(31,1 \%)$ das unidades participantes. Na RPS foram excluídas as unidades de pronto-atendimento, o hospital municipal e os ambulatórios de saúde mental adulto e infantil.

Um grupo de 35 detentos $(24,8 \%)$ relatou que a última prisão foi há menos de um ano e 11 (7,8\%) há mais de seis anos (dados não apresentados em tabela). Na Tabela 1 está mostrado que os detentos e funcionários do presídio foram diferentes nas variáveis idade, cor, sexo e anos de estudo ( $p<0,001)$. Entre os funcionários do presídio e da RPS as diferenças foram em relação à idade, sexo, anos de trabalho $(\mathrm{p}<0.001)$ e anos de estudo ( $p=0,041$ ).

$\mathrm{Na}$ investigação de sintomas respiratórios, os detentos apresentaram tosse com escarro numa proporção maior que os funcionários do presídio ( $15,6 \%$ vs $2,6 \%$, $\mathrm{p}=$ 0,0012). Também a afirmação de já ter feito tratamento para a doença foi maior entre os detentos $(13,5 \%$ vs $2,6 \%, p=0,007)$.

\section{Conhecimentos}

Os funcionários do presídio referiram ter recebido informações sobre TB numa proporção maior que os da RPS ( $63,5 \%$ vs $29,8 \%$, $\mathrm{p}<0,001$ ) (dados não apresentados em tabela). Segundo a Tabela 2, houve diferenças significativas quanto ao conhecimento dos sintomas entre detentos e funcionários do presídio e entre estes e os da RPS. Para os detentos, a TB pode ser transmitida pelo ar $(49,6 \%)$, pelo compartilhamento de cigarros $(12,1 \%)$ e pelos talheres $(10,6 \%)$, e as formas de contágio eram desconhecidas por $22,0 \%$. Funcionários do presídio $(44,3 \%)$ e RPS $(39,9 \%)$ referiram a transmissão através do compartilhamento de pratos e talheres.

Quando perguntado "como uma pessoa pode se prevenir da TB", 38,3\% dos detentos não souberam responder. Para $40,9 \%$ dos funcionários do presídio e 46,8\% da RPS, uma das respostas foi "não compartilhar pratos e talheres" (Tabela 3).

A TB foi considerada uma doença curável para as três categorias estudadas. A diferença foi verificada no item "como se cura a TB", quando a alternativa "tratamento com supervisão médica" foi respondida por $36,9 \%$ dos detentos e 75,6\% dos funcionários do presídio $(\mathrm{p}<0.001$ ). Apenas $24,1 \%$ dos detentos disseram que o tratamento para TB é gratuito. Entre os funcionários do presídio foram $58,3 \%$, e na RPS $84,8 \%$ ( $\mathrm{p}<$ 0.001) (Tabela 3).

\section{Comportamento e atitudes diante da possibilidade de contrair a tuberculose}

A Tabela 4 mostra que, para $24,7 \%$ dos funcionários da RPS, o contato com pessoas em local fechado é considerado fator favorável à contaminação. Entre estes funcionários, 22,1\% não souberam responder "por que pode pegar TB".

No roteiro KAP, a pergunta "o que 
Tabela 1 - Características sociodemográficas e variáveis relacionadas à tuberculose entre detentos, funcionários de unidade prisional e rede pública de saúde. Hortolândia, 2010.

Table 1 - Sociodemographic characteristics and variables related to tuberculosis between prisoners, prisional unit employees and public health workers. Hortolândia, 2010.

\begin{tabular}{|c|c|c|c|c|c|c|c|c|}
\hline \multirow{3}{*}{$\begin{array}{l}\text { Características sociodemográficas e } \\
\text { variáveis relacionadas à TB }\end{array}$} & \multicolumn{2}{|c|}{ Detentos } & \multicolumn{2}{|c|}{$\begin{array}{l}\text { Funcionários do } \\
\text { presídio }\end{array}$} & \multicolumn{2}{|c|}{$\begin{array}{l}\text { Funcionários da } \\
\text { rede pública de } \\
\text { saúde }\end{array}$} & \multirow[b]{3}{*}{ valor $\mathrm{p}^{\mathrm{a}}$} & \multirow[b]{3}{*}{ valor $\mathrm{p}^{\mathrm{b}}$} \\
\hline & \multicolumn{2}{|c|}{$\mathrm{N}=141$} & \multicolumn{2}{|c|}{$\mathrm{N}=115$} & \multicolumn{2}{|c|}{$\mathrm{N}=158$} & & \\
\hline & $\mathrm{N}$ & $\%$ & & $\%$ & $\mathrm{~N}$ & $\%$ & & \\
\hline Idade & & & & & & & $<0,001$ & 0,005 \\
\hline $18-29$ & 83 & 58,9 & 20 & 17,4 & 31 & 19,6 & & \\
\hline $30-39$ & 47 & 33,3 & 51 & 44,4 & 42 & 26,6 & & \\
\hline $40-49$ & 9 & 6,4 & 32 & 27,8 & 48 & 30,4 & & \\
\hline$\geq 50$ & 2 & 1,4 & 12 & 10,4 & 37 & 23,4 & & \\
\hline Cor & & & & & & & $<0,001$ & 0,161 \\
\hline Branco & 75 & 53,2 & 91 & 79,1 & 112 & 70,9 & & \\
\hline Não Branco & 66 & 46,8 & 24 & 20,9 & 46 & 29,1 & & \\
\hline Sexo & & & & & & & $<0,001$ & $<0,001$ \\
\hline Masculino & 141 & 100,0 & 92 & 80,0 & 33 & 20,9 & & \\
\hline Feminino & - & - & 23 & 20,0 & 125 & 79,1 & & \\
\hline Anos de estudo & & & & & & & $<0,001$ & 0,041 \\
\hline $0-4$ & 31 & 22,0 & - & - & & & & \\
\hline 5- 8 & 74 & 52,5 & - & - & 9 & 5,7 & & \\
\hline $9-11$ & 34 & 24,1 & 51 & 44,3 & 64 & 40,5 & & \\
\hline $12-16$ & 2 & 1,4 & 54 & 47,0 & 65 & 41,1 & & \\
\hline$\geq 17$ & - & - & 10 & 8,7 & 20 & 12,7 & & \\
\hline Anos de trabalho & & & & & & & - & $<0,001$ \\
\hline$<1$ & - & - & 10 & 8,7 & 50 & 31,6 & & \\
\hline $1-9$ & - & - & 69 & 60,0 & 80 & 50,6 & & \\
\hline $10-19$ & - & - & 31 & 27,0 & 26 & 16,5 & & \\
\hline$\geq 20$ & - & - & 5 & 4,3 & 2 & 1,3 & & \\
\hline Tem tosse com catarro & & & & & & & 0,0012 & 0,627 \\
\hline Sim & 22 & 15,6 & 3 & 2,6 & 4 & 2,5 & & \\
\hline Não & 119 & 84,4 & 112 & 97,4 & 154 & 97,5 & & \\
\hline Já tratou TB ou está em tratamento & & & & & & & 0,007 & 0,202 \\
\hline Sim & 19 & 13,5 & 3 & 2,6 & 1 & 0,6 & & \\
\hline Não & 120 & 85,1 & 111 & 96,5 & 157 & 99,4 & & \\
\hline Em tratamento & 2 & 1,4 & 1 & 0,9 & - & - & & \\
\hline
\end{tabular}

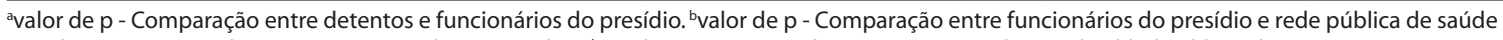
${ }^{a} p$-value -Comparison between inmates and prison workers. ${ }^{b} p$-value -Comparison between prison workers and public health workers

sentiria se tivesse TB" propicia conhecer o sentimento despertado pela doença. Para os detentos, foi tristeza (39,7\%) e preocupação (16,3\%). Entre os funcionários do presídio, tristeza (41,7\%) e medo (26\%), e entre aqueles da RSP medo $(25,9 \%)$ e surpresa $(22,8 \%)$.

Perguntados se falariam sobre a doença, todos os detentos e funcionários do presídio responderam afirmativamente. Na RPS foram $135(85,4 \%)(\mathrm{p}<0,001)$ (dados não mostrados em tabela).

No grupo de detentos, a maior preocupação quando se pensa em TB é ficar doente e o acesso ao tratamento e cura. A morte foi citada por $12,8 \%$ dos entrevistados deste grupo. 
Tabela 2 - Conhecimento sobre a tuberculose (Parte I) entre detentos, funcionários de unidade prisional e rede pública de saúde. Hortolândia, 2010.

Table 2 - Knowlwdge about tuberculosis (Part I) between prisoners, prisional unit employees and public health workers.

Hortolândia, 2010.

\begin{tabular}{|c|c|c|c|c|c|c|c|c|}
\hline \multirow[t]{3}{*}{ Conhecimento sobre a TB (Parte I) } & \multicolumn{2}{|c|}{ Detentos } & \multicolumn{2}{|c|}{$\begin{array}{c}\text { Funcionários do } \\
\text { presídio }\end{array}$} & \multicolumn{2}{|c|}{$\begin{array}{l}\text { Funcionários da } \\
\text { rede pública de } \\
\text { saúde }\end{array}$} & \multirow[b]{3}{*}{ valor $p^{a}$} & \multirow[b]{3}{*}{ valor $\mathrm{p}^{\mathrm{b}}$} \\
\hline & \multicolumn{2}{|c|}{$\mathrm{N}=141$} & \multicolumn{2}{|c|}{$\mathrm{N}=115$} & \multicolumn{2}{|c|}{$\mathrm{N}=158$} & & \\
\hline & $\mathrm{N}$ & $\%$ & $\mathrm{~N}$ & $\%$ & $\mathrm{~N}$ & $\%$ & & \\
\hline Opinião sobre a TB & & & & & & & 0,140 & 0,354 \\
\hline Muito grave & 53 & 37,6 & 51 & 44,4 & 72 & 45,6 & & \\
\hline Grave & 83 & 58,9 & 61 & 53,0 & 83 & 52,5 & & \\
\hline Não é grave & 5 & 3,5 & 1 & 0,9 & 3 & 1,9 & & \\
\hline Não sabe & - & - & 2 & 1,7 & - & - & & \\
\hline \multicolumn{9}{|l|}{ Sintomas da TB ${ }^{c}$} \\
\hline Tosse seca & 89 & 63,1 & 73 & 63,5 & 56 & 35,4 & 0,953 & $<0,001$ \\
\hline Tosse + de 2 semanas & 7 & 5,0 & 76 & 66,1 & 137 & 86,7 & $<0,001$ & $<0,001$ \\
\hline Tosse com catarro & 40 & 28,4 & 67 & 58,3 & 83 & 52,5 & $<0,001$ & 0,381 \\
\hline Tosse com sangue & 26 & 18,4 & 64 & 55,6 & 89 & 56,3 & $<0,001$ & 0,911 \\
\hline Febre & 36 & 25,5 & 60 & 52,2 & 63 & 39,9 & $<0,001$ & 0,044 \\
\hline Febre sem causa por mais de 7 dias & - & - & 39 & 33,9 & 59 & 37,3 & $<0,001$ & 0,560 \\
\hline Dor de cabeça & 17 & 12,1 & 18 & 15,6 & 11 & 7,0 & 0,405 & 0,021 \\
\hline Cansaço & 32 & 22,7 & 56 & 48,7 & 82 & 51,9 & $<0,001$ & 0,601 \\
\hline Náusea & 1 & 0,7 & 18 & 15,6 & 10 & 6,3 & $<0,001$ & 0,012 \\
\hline Dor no peito & 35 & 24,8 & 57 & 49,6 & 48 & 30,4 & $<0,001$ & 0,0013 \\
\hline Perda de peso & 14 & 9,9 & 59 & 51,3 & 107 & 67,7 & $<0,001$ & 0,006 \\
\hline Falta de ar & 6 & 4,3 & 51 & 44,4 & 69 & 43,7 & $<0,001$ & 0,911 \\
\hline Suor noturno & 23 & 16,3 & 6 & 5,2 & - & - & 0,005 & 0,013 \\
\hline Falta de apetite & 13 & 9,2 & 1 & 0,9 & - & - & 0,003 & - \\
\hline Não sabe & 13 & 9,2 & 4 & 3,5 & 2 & 1,3 & 0,067 & 0,416 \\
\hline Outros ${ }^{c}$ & 11 & 7,8 & 3 & 2,6 & 2 & 1,3 & 0,069 & 0,719 \\
\hline \multicolumn{9}{|l|}{ Como se pega TB ${ }^{d}$} \\
\hline Através de aperto de mão & 9 & 6,4 & 11 & 9,6 & 5 & 3,2 & 0,345 & 0,026 \\
\hline Através do ar & 70 & 49,6 & 102 & 88,7 & 150 & 94,9 & $<0,001$ & 0,056 \\
\hline Compartilhando pratos e talheres & 15 & 10,6 & 51 & 44,3 & 63 & 39,9 & $<0,001$ & 0,459 \\
\hline Comendo no mesmo prato & 1 & 0,7 & 40 & 34,8 & 39 & 24,7 & $<0,001$ & 0,069 \\
\hline Compartilhando cigarros & 17 & 12,1 & 2 & 1,8 & - & - & 0,002 & - \\
\hline Banho frio/chão gelado & 12 & 8,5 & - & - & - & - & 0,001 & - \\
\hline Contato com o doente com TB & 6 & 4,3 & 9 & 7,8 & - & - & 0,226 & 0,273 \\
\hline Pela saliva & 5 & 3,6 & 1 & 0,9 & 2 & 1,3 & 0,321 & 0,781 \\
\hline Tocando em maçanetas & - & - & 14 & 12,2 & 13 & 8,2 & $<0,001$ & 0,281 \\
\hline Não sabe & 31 & 22,0 & 6 & 5,2 & 4 & 2,5 & $<0,001$ & 0,401 \\
\hline Outros $^{d}$ & 6 & 4,3 & 4 & 3,5 & 2 & 1,3 & 0,996 & 0,416 \\
\hline
\end{tabular}

a valor de $\mathrm{p}$ - Comparação entre detentos e funcionários do presídio. ${ }^{\mathrm{b}}$ valor de $\mathrm{p}$ - Comparação entre funcionários do presídio e rede pública de saúde. As variáveis c, d permitiram mais de uma resposta. Outros (c) Diarréia, dor abdominal, vômitos, gripe, tontura, gânglios, impotência, depressão. Outros (d) Uso de drogas, beijo, alimentos contaminados.

${ }^{a} p$-value -Comparison between inmates and prison workers. ${ }^{b} p$-value - Comparison between prison workers and public health workers. Variables $c$ and $d$ allowed for more than one response. Others (c) Diarrhea, abdominal pains, vomiting, flu, dizziness, ganglia, impotence, depression. Others (d) Drug use, kissing, contaminated food 
Tabela 3 - Conhecimento sobre a tuberculose (Parte II) entre detentos, funcionários de unidade prisional e rede pública de saúde. Hortolândia, 2010.

Table 3 - Knowledge about tuberculosis (Part II) between prisoners, prisional unit employees and public health workers.

Hortolândia, 2010.

\begin{tabular}{|c|c|c|c|c|c|c|c|c|}
\hline \multirow[t]{3}{*}{ Conhecimento sobre a TB (Parte II) } & \multicolumn{2}{|c|}{ Detentos } & \multicolumn{2}{|c|}{$\begin{array}{l}\text { Funcionários do } \\
\text { presídio }\end{array}$} & \multicolumn{2}{|c|}{$\begin{array}{l}\text { Funcionários da } \\
\text { rede pública de } \\
\text { saúde }\end{array}$} & \multirow[b]{3}{*}{ valor $p^{a}$} & \multirow[b]{3}{*}{ valor $\mathrm{p}^{\mathrm{b}}$} \\
\hline & \multicolumn{2}{|c|}{$\mathrm{N}=141$} & \multicolumn{2}{|c|}{$\mathrm{N}=115$} & \multicolumn{2}{|c|}{$\mathrm{N}=158$} & & \\
\hline & $\mathrm{N}$ & $\%$ & $\mathrm{~N}$ & $\%$ & $\mathrm{~N}$ & $\%$ & & \\
\hline \multicolumn{9}{|l|}{ Como a pessoa pode se prevenir da TBc } \\
\hline Evitar cumprimentar com as mãos & 2 & 1,4 & 7 & 6,1 & 9 & 5,7 & 0,094 & 0,892 \\
\hline Cobrir a boca ao tossir ou espirrar & 5 & 3,6 & 61 & 53,0 & 129 & 81,6 & $<0,001$ & $<0,001$ \\
\hline Evitar compartilhar pratos, talheres & 15 & 10,6 & 47 & 40,9 & 74 & 46,8 & $<0,001$ & 0,327 \\
\hline Lavar as mãos, boa higiene & 7 & 5,0 & 48 & 41,7 & 66 & 41,8 & $<0,001$ & 0,996 \\
\hline Fechar as janelas & - & - & 7 & 6,1 & 2 & 1,3 & 0,010 & 0,063 \\
\hline Alimentar-se bem & 5 & 3,5 & 54 & 47,0 & 59 & 37,3 & $<0,001$ & 0,111 \\
\hline Evitar o doente & 24 & 17,0 & 13 & 11,3 & 1 & 0,6 & 0,196 & $<0,001$ \\
\hline Evitar o frio & 12 & 8,5 & - & - & - & - & 0,0013 & - \\
\hline Evitar lugar fechado/não ventilado & 7 & 5,0 & 12 & 10,4 & 3 & 1,9 & 0,097 & 0,002 \\
\hline Evitar compartilhar cigarro & 6 & 4,3 & - & - & - & - & 0,068 & - \\
\hline Isolar o doente/usar máscara & 7 & 5,0 & 11 & 9,6 & - & - & 0,152 & $<0,001$ \\
\hline Medicação & 6 & 4,3 & - & - & - & - & 0,068 & - \\
\hline Não se previne & 6 & 4,3 & - & - & - & - & 0,068 & - \\
\hline Orar, rezar & - & - & 7 & 6,1 & 1 & 0,6 & 0,010 & 0,023 \\
\hline Não sabe & 54 & 38,3 & 12 & 10,4 & 3 & 1,9 & $<0,001$ & 0,002 \\
\hline Outros $^{c}$ & 8 & 5,7 & 10 & 8,7 & 2 & 1,3 & 0,347 & 0,003 \\
\hline \multicolumn{9}{|l|}{ Quem pode pegar TB ${ }^{d}$} \\
\hline Qualquer pessoa & 98 & 69,5 & 100 & 87,0 & 157 & 99,4 & $<0,001$ & $<0,001$ \\
\hline $\begin{array}{l}\text { Apenas pessoas pobres/ morador } \\
\text { rua/ uso álcool/drogas/ com HIV/ } \\
\text { apenas nas prisões }\end{array}$ & 5 & 3,6 & 3 & 2,6 & 7 & 4,0 & 0,946 & 0,841 \\
\hline Fumantes & 6 & 4,3 & 2 & 1,7 & - & - & 0,430 & - \\
\hline Pessoas debilitadas & 11 & 7,8 & 6 & 5,2 & 1 & 0,6 & 0,409 & 0,048 \\
\hline $\begin{array}{l}\text { Pessoas que têm contato com o } \\
\text { doente }\end{array}$ & 6 & 4,3 & 4 & 3,5 & - & - & 0,996 & 0,064 \\
\hline Não sabe & 10 & 7,1 & 1 & 0,9 & - & - & 0,033 & - \\
\hline Outros $^{d}$ & 5 & 3,6 & 4 & 3,5 & - & - & 0,755 & - \\
\hline \multicolumn{9}{|l|}{ Como se cura a TB e } \\
\hline Medicamentos do posto de saúde & 104 & 73,8 & 86 & 74,8 & 106 & 67,1 & 0,852 & 0,169 \\
\hline Tratamento com supervisão médica & 52 & 36,9 & 87 & 75,6 & 128 & 81,0 & $<0,001$ & 0,285 \\
\hline Isolamento do doente & 8 & 5,7 & 1 & 0,9 & - & - & 0,083 & - \\
\hline Orações & 5 & 3,6 & 4 & 3,5 & 2 & 1,3 & 0,755 & 0,416 \\
\hline Não sabe & 6 & 4,3 & 4 & 3,5 & 2 & 1,3 & 0,996 & 0,416 \\
\hline Outros $\mathrm{e}$ & 1 & 0,7 & 6 & 5,2 & 4 & 2,5 & 0,070 & 0,40 \\
\hline Quanto custa o tratamento da TB & & & & & & & $<0,001$ & $<0,001$ \\
\hline É de graça & 34 & 24,1 & 67 & 58,3 & 134 & 84,8 & & \\
\hline É caro & 23 & 16,3 & 17 & 14,8 & 6 & 3,8 & & \\
\hline Não sabe & 84 & 59,6 & 31 & 26,9 & 18 & 11,4 & & \\
\hline
\end{tabular}

${ }^{a}$ valor de $\mathrm{p}$ - Comparação entre detentos e funcionários do presídio. ${ }^{\mathrm{b}}$ valor de $\mathrm{p}$ - Comparação entre funcionários do presídio e rede pública de saúde. As variáveis c, d, e permitiram mais de uma resposta. Outros (c) Evitar bebidas alcoólicas e cigarro, evitar poeira, tomar vacina, fazer exames periódicos/atividade física. Outros (d) Quem não se previne, idosos, crianças. Outros (e) Não tem cura, ervas medicinais, repouso, ventilação.

${ }^{a} p$-value -Comparison between inmates and prison workers. ${ }^{b} p$-value - Comparison between prison workers and public health workers. Variables $c, d$, and e allowed more than one response. Others (c) Avoid alcoholic drinks and cigarettes, avoid dust, be vaccinated, have routine exams/physical activity. Others (d) Who do not need to prevent, seniors, children. Others (e) There is no cure, medicinal herbs, rest, ventilation 
Tabela 4 - Comportamento e atitudes, diante da possibilidade de contrair a tuberculose, entre detentos e funcionários de unidade prisional e rede pública de saúde. Hortolândia, 2010.

Table 4 - Behavior and attitudes between prisoners, prisional unit employees and public health workers in front of the possibility to catch tuberculosis. Hortolândia, 2010.

\begin{tabular}{|c|c|c|c|c|c|c|c|c|}
\hline \multirow[t]{3}{*}{ Comportamento e atitudes } & \multicolumn{2}{|c|}{ Detentos } & \multicolumn{2}{|c|}{$\begin{array}{c}\text { Funcionários do } \\
\text { presídio }\end{array}$} & \multicolumn{2}{|c|}{$\begin{array}{l}\text { Funcionários da } \\
\text { rede pública de } \\
\text { saúde }\end{array}$} & \multirow[b]{3}{*}{ valor $\mathrm{p}^{\mathrm{a}}$} & \multirow[b]{3}{*}{ valor $\mathrm{p}^{\mathrm{b}}$} \\
\hline & \multicolumn{2}{|c|}{$\mathrm{N}=141$} & \multicolumn{2}{|c|}{$\mathrm{N}=115$} & \multicolumn{2}{|c|}{$\mathrm{N}=158$} & & \\
\hline & $\mathrm{N}$ & $\%$ & $\mathrm{~N}$ & $\%$ & $\mathrm{~N}$ & $\%$ & & \\
\hline \multicolumn{9}{|l|}{ Por que pode pegar TB ${ }^{c}$} \\
\hline Convivo com doentes de TB & 36 & 25,5 & 25 & 21,7 & 21 & 13,3 & 0,479 & 0,066 \\
\hline Contato com pessoas em local fechado & 21 & 14,9 & 27 & 23,5 & 39 & 24,7 & 0,080 & 0,818 \\
\hline Ambiente desfavorável/friagem/fumo & 22 & 15,6 & 10 & 8,7 & - & - & 0,096 & $<0,001$ \\
\hline Local de trabalho & - & - & 28 & 24,3 & 10 & 6,3 & $<0,001$ & $<0,001$ \\
\hline Exposto pelo ar & 12 & 8,5 & 6 & 5,2 & 6 & 3,8 & 0,305 & $<0,001$ \\
\hline Qualquer um pode pegar & 35 & 24,8 & 15 & 13,0 & 36 & 22,8 & 0,018 & $<0,001$ \\
\hline Não me previno & 4 & 2,8 & 3 & 2,6 & 1 & 0,6 & 0,784 & 0,406 \\
\hline Não sabe & 6 & 4,3 & 3 & 2,6 & 35 & 22,1 & 0,711 & $<0,001$ \\
\hline Outros $^{c}$ & 7 & 5,0 & - & - & 7 & 4,4 & 0,041 & 0,058 \\
\hline \multicolumn{9}{|l|}{$O$ que sentiria se tivesse $T^{d}{ }^{d}$} \\
\hline Medo & 15 & 10,6 & 30 & 26,1 & 41 & 25,9 & 0,0012 & 0,980 \\
\hline Surpresa & 13 & 9,2 & 28 & 24,3 & 36 & 22,8 & 0,0010 & 0,763 \\
\hline Vergonha & - & - & 3 & 2,6 & 5 & 3,2 & 0,178 & 0,925 \\
\hline Constrangimento & 6 & 4.3 & 17 & 14,8 & 13 & 8,2 & 0,003 & 0,087 \\
\hline Desespero & 5 & 3.5 & 15 & 13,0 & 9 & 5,7 & 0,005 & 0,034 \\
\hline Tristeza & 56 & 39,7 & 48 & 41,7 & 35 & 22,1 & 0,743 & $<0,001$ \\
\hline Preocupação & 23 & 16,3 & 17 & 14,8 & 5 & 3,2 & 0,737 & $<0,001$ \\
\hline Ficaria "normal" & 7 & 5,0 & - & - & 1 & 0,6 & 0,042 & - \\
\hline Não sentiria nada & 15 & 10,6 & 20 & 17,4 & 26 & 16,5 & 0,118 & 0,838 \\
\hline Não sabe & 10 & 7,1 & 6 & 5,2 & 21 & 13,3 & 0,538 & 0,027 \\
\hline Outros $^{d}$ & 2 & 1,4 & 5 & 4,3 & 4 & 2,5 & 0,296 & 0,627 \\
\hline \multicolumn{9}{|l|}{ Para quem falaria e } \\
\hline Médico & 25 & 17,7 & 72 & 62,6 & 143 & 90,5 & $<0,001$ & $<0,001$ \\
\hline Esposa(o) & 13 & 9,2 & 81 & 70,4 & 103 & 65,2 & $<0,001$ & 0,361 \\
\hline Pais & 10 & 7,1 & 85 & 73,9 & 93 & 58,9 & $<0,001$ & 0,010 \\
\hline Filhos & 6 & 4,3 & 78 & 67,8 & 95 & 60,1 & $<0,001$ & 0,192 \\
\hline Outro membro da família & 17 & 12,1 & 68 & 59,1 & 62 & 39,2 & $<0,001$ & 0,0012 \\
\hline Amigo & 71 & 50,4 & 87 & 75,6 & 74 & 46,8 & $<0,001$ & $<0,001$ \\
\hline Pessoas próximas & 23 & 16,3 & 22 & 19,1 & 2 & 1,3 & 0,556 & $<0,001$ \\
\hline Qualquer pessoa & 30 & 21,3 & 12 & 10,4 & 1 & 0,6 & 0,020 & $<0,001$ \\
\hline Não sabe & - & - & 3 & 2,6 & 3 & 1,9 & 0,178 & 0,982 \\
\hline Outros $^{e}$ & 2 & 1,4 & - & - & 1 & 0,6 & - & - \\
\hline \multicolumn{9}{|l|}{$\begin{array}{l}\text { O que faria se achasse que tem } \\
\text { sintomas da TB }\end{array}$} \\
\hline Procuraria um posto de atendimento & 134 & 95,0 & 111 & 96,5 & 157 & 99,4 & 0,784 & 0,099 \\
\hline Faria tratamento próprio (ervas, etc) & 1 & 0,7 & 1 & 0,9 & 8 & 5,1 & - & 0,116 \\
\hline Não sabe & 2 & 1,4 & 2 & 1,7 & 1 & 0,6 & 0,764 & 0,781 \\
\hline Outros ${ }^{f}$ & 3 & 2,1 & 2 & 1,7 & 2 & 1,3 & 0,818 & 0,850 \\
\hline
\end{tabular}


Tabela 4 - Comportamento e atitudes, diante da possibilidade de contrair a tuberculose, entre detentos e funcionários de unidade prisional e rede pública de saúde. Hortolândia, 2010. (continuação)

Table 4 - Behavior and attitudes between prisoners, prisional unit employees and public health workers in front of the possibility to catch tuberculosis. Hortolândia, 2010. (continuation)

\begin{tabular}{|c|c|c|c|c|c|c|c|c|}
\hline \multirow[t]{3}{*}{ Comportamento e atitudes } & \multicolumn{2}{|c|}{ Detentos } & \multicolumn{2}{|c|}{$\begin{array}{l}\text { Funcionários do } \\
\text { presídio }\end{array}$} & \multicolumn{2}{|c|}{$\begin{array}{l}\text { Funcionários da } \\
\text { rede pública de } \\
\text { saúde }\end{array}$} & \multirow[b]{3}{*}{ valor $\mathrm{p}^{\mathrm{a}}$} & \multirow[b]{3}{*}{ valor $p$} \\
\hline & \multicolumn{2}{|c|}{$\mathrm{N}=141$} & \multicolumn{2}{|c|}{$\mathrm{N}=115$} & \multicolumn{2}{|c|}{$\mathrm{N}=158$} & & \\
\hline & $\mathrm{N}$ & $\%$ & $\mathrm{~N}$ & $\%$ & $\mathrm{~N}$ & $\%$ & & \\
\hline $\begin{array}{l}\text { Em que momento procuraria o posto } \\
\text { de atendimento }\end{array}$ & & & & & & & $<0,001$ & $<0,001$ \\
\hline $\begin{array}{l}\text { Quando os sintomas persistirem por } \\
\text { mais de } 15 \text { dias }\end{array}$ & 10 & 7,1 & 33 & 28,7 & 92 & 58,2 & & \\
\hline $\begin{array}{l}\text { Assim que perceber que os sintomas } \\
\text { são da TB }\end{array}$ & 119 & 84,4 & 80 & 69,6 & 57 & 36,1 & & \\
\hline Não sabe & 5 & 3,5 & 2 & 1,7 & 8 & 5,1 & & \\
\hline Outros 9 & 7 & 5,0 & - & - & 1 & 0,6 & & \\
\hline $\begin{array}{l}\text { Maior preocupação quando pensa em } \\
\text { TB }^{h}\end{array}$ & & & & & & & & \\
\hline Acesso ao tratamento / Cura & 29 & 20,6 & 25 & 21,7 & 44 & 27,8 & 0,819 & 0,251 \\
\hline Passar a doença para a família & 21 & 14,9 & 23 & 20,0 & 14 & 8,9 & 0,281 & 0,008 \\
\hline Contágio & 17 & 12,1 & 18 & 15,6 & 25 & 15,8 & 0,405 & 0,969 \\
\hline Ficar doente & 29 & 20,6 & 18 & 15,6 & 15 & 9,5 & 0,312 & 0,123 \\
\hline Morte & 18 & 12,8 & 6 & 5,2 & 4 & 2,5 & 0,393 & 0,401 \\
\hline Manter-se com saúde & 17 & 12,1 & 10 & 8,7 & 10 & 6,3 & 0,384 & 0,459 \\
\hline Falta da informação sobre TB & - & - & - & - & 14 & 8,9 & - & 0,0011 \\
\hline Não pensa & 9 & 6,4 & 7 & 6,1 & 5 & 3,2 & 0,922 & 0,245 \\
\hline Não sabe & 1 & 0,7 & 2 & 1,7 & 26 & 16,5 & 0,859 & $<0,001$ \\
\hline Outros ${ }^{\mathbf{h}}$ & 2 & 1,4 & 13 & 11,3 & 3 & 1,9 & $<0,001$ & 0,0011 \\
\hline
\end{tabular}

a valor de $\mathrm{p}$ - Comparação entre detentos e funcionários do presídio. ${ }^{\mathrm{b}}$ valor de $\mathrm{p}$ - Comparação entre funcionários do presídio e rede pública de saúde. As variáveis $c, d, f, h$ permitiram mais de uma resposta. Outro (c) Já tive TB, usei drogas, Deus pode querer. Outro (d) Estou predestinado, enfrentaria, procuraria tratamento. Outro (e) Falaria com Deus, falaria com quem confio, não falaria para ninguém. Outro (f) Pararia de fumar, procuraria mais informações, procuraria farmácia, procuraria Deus. Outro (g) Quando tratamento por conta não funcionasse, quando estivesse fraco, não iria ao posto. Outro (h) TB-MDR, estar doente e não saber, isolamento, epidemia.

${ }^{a} p$-value - Comparison between inmates and prison workers. ${ }^{b} p$-value - Comparison between prison workers and public health workers. Variables $c, d, f$ and $h$ allowed for more than one response. Other (c) I already had TB, took drugs, God might want this. Other (d) I am predestined, I would face it, find treatment. Other (e) I would talk to God, talk with somebody I trust, would not talk to anybody. Other (f) Would stop smoking, obtain more information, go to a drugstore, go to God. Other $(g)$ When self-treatment did not work, when you are weak, would not go to a health center. Other (h) TB-MDR, to be sick and not to know it, isolation, epidemic

\section{Atitudes em relação a doentes com tuberculose}

Sobre "como um doente de TB é considerado pelas outras pessoas" (Tabela 5), $41,1 \%$ dos detentos declararam que "muitos rejeitam”. Entre funcionários do presídio foram $25,2 \%$ e na RPS $22,8 \%$ os que referiram que "muitos são amigáveis, mas tentam evitá-las".

Ao serem questionados por que uma pessoa vivendo com HIV também pode ter tuberculose, $44,3 \%$ dos funcionários do presídio citaram a baixa imunidade, proporção inferior à encontrada na RPS $(61,4 \%)(p=0,005)$. Chama-se a atenção para a proporção de funcionários da RPS que responderam a alternativa "não sabe" (21,5\%).

\section{Discussão}

O aumento do uso de drogas foi um dos fatores que contribuiu para o crescimento da violência no país e consequentemente o aumento da população prisional. ${ }^{15} \mathrm{Os}$ detentos provêm de classes sociais determinadas pela pobreza, com dificuldades de 
Tabela 5 - Atitudes em relação a doentes com TB, de detentos e funcionários de unidade prisional e da rede pública de saúde. Hortolândia, 2010.

Table 5 - Attitudes from prisoners, prisional unit employees and public health workers related to sick people with TB.

Hortolândia, 2010.

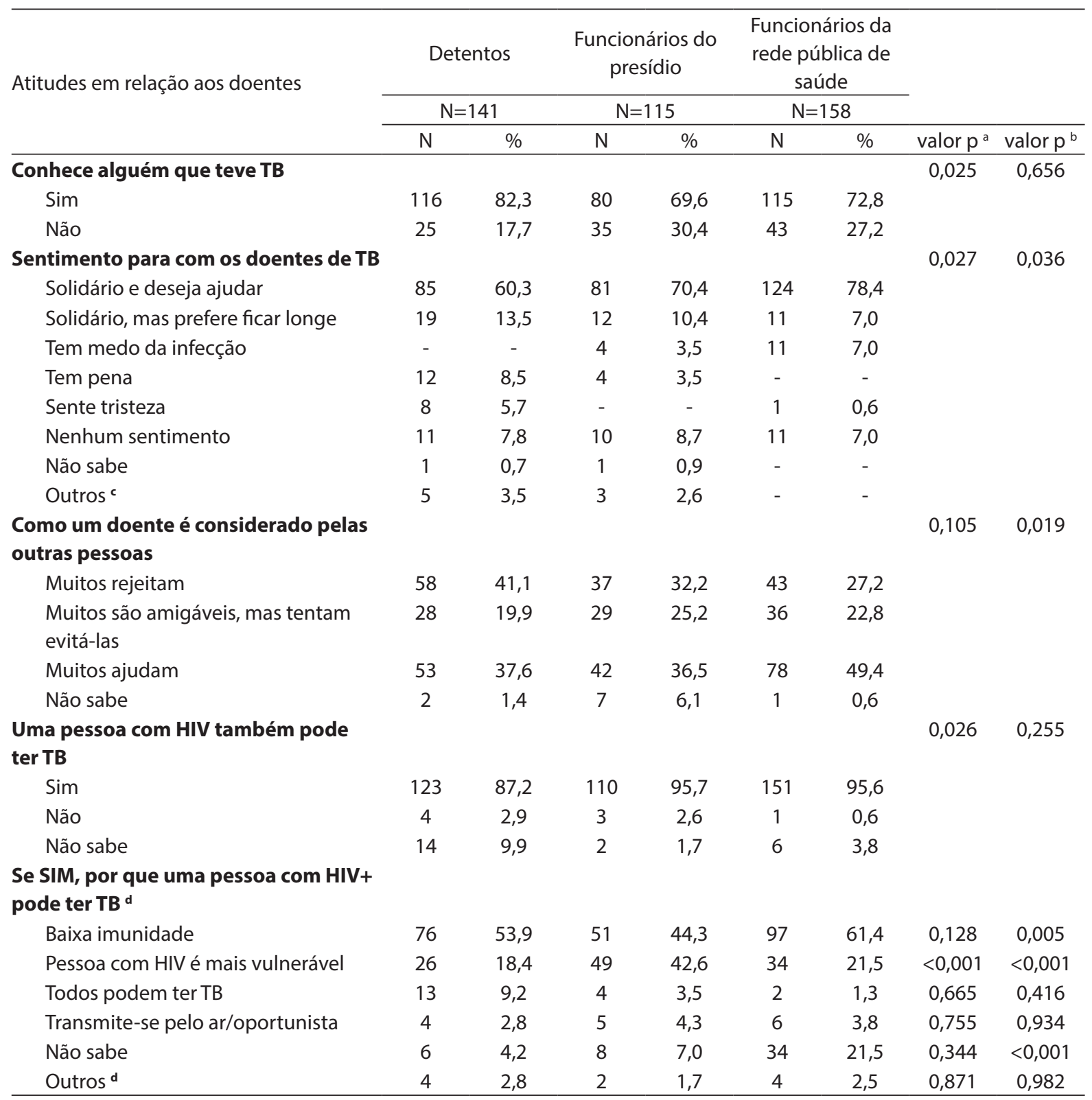

a valor de $\mathrm{p}$ - Comparação entre detentos e funcionários do presídio. ${ }^{\mathrm{b}}$ valor de $\mathrm{p}$ - Comparação entre funcionários do presídio e rede pública de saúde. A variável d permitiu mais de uma resposta. Outro (c) É problema deles, perda, discriminação, raiva. Outro (d) Já toma medicamentos, não se previne, risco para HIV.

${ }^{a} p$-value - Comparison between inmates and prison workers. ${ }^{b} p$-value - Comparison between prison workers and public health workers. Variable $d$ allowed for more than one response. Other (c) It is their problem, loss, discrimination, anger. Other (d) I already take the medicine, do nothing to prevent, risk of HIV

acesso à saúde, à educação, à informação e com baixa escolaridade, características também encontradas nas populações mais atingidas pela TB. Estas condições são favoráveis à perpetuação das crenças a respeito da doença e ao desconhecimento sobre a sua existência, formas de transmissão, infecção e controle.

Neste estudo, a população de detentos era formada por indivíduos brancos (53,2\%), 
semelhante para a cidade de São Paulo, ${ }^{16}$ e $22 \%$ estudaram menos de cinco anos. Segundo o Plano Nacional de Saúde no Sistema Prisional, ${ }^{17}$ a população penitenciária brasileira é composta por homens brancos, solteiros e com menos de 30 anos de idade, poucos foram alfabetizados e sem profissão definida anteriormente à prisão, caracterizando uma situação de exclusão social anterior ao seu ingresso no Sistema Prisional. Assim, é possível considerar que a população penitenciária estudada em Hortolândia tem uma condição de escolaridade superior à media nacional, visto que apenas $22 \%$ tinham menos que cinco anos de estudo.

Sabe-se que o conhecimento pode influenciar as práticas em relação à prevenção. ${ }^{18}$ Grande parte dos detentos e funcionários do presídio não recebeu informações sobre a TB e houve um considerável número de respostas erradas sobre a prevenção destas doenças, semelhantes ao de outros autores, ${ }^{19}$ sugerindo que as formas de comunicação, bem como as estratégias utilizadas no repasse do conhecimento no presídio, não atingem seus objetivos e demonstram a ausência da instituição penitenciária nas intervenções de educação em saúde e na sua contribuição para o controle da TB. ${ }^{20}$

Na análise do conhecimento da população brasileira sobre $\mathrm{TB},{ }^{21} 34 \%$ dos entrevistados afirmaram conhecer alguém que teve ou tem a doença. Entre os detentos, $82,3 \% \mathrm{e}$ $69,6 \%$ dos funcionários do presídio responderam afirmativamente a esta questão, sugerindo familiaridade com a doença, porém o conhecimento é permeado por crenças e informações equivocadas. Na RPS, apesar de melhor desempenho sobre conhecimento sobre a TB, foram observados vários erros conceituais básicos quando a questão se referia à vulnerabilidade no compartilhamento de objetos. Resultados similares aparecem em estudos com trabalhadores de enfermagem ${ }^{9}$ e com equipes de saúde da família; ${ }^{22}$ entretanto, estas informações são facilmente encontradas em manuais e cartilhas disponíveis na Internet e nos departamentos de vigilância epidemiológica, ${ }^{23}$ indicando deficiências nas ações de educação na RPS e a necessidade da socialização das informações sobre TB para os demais profissionais, numa abordagem multidisciplinar e intersetorial. ${ }^{9,10}$ Os treinamentos são estratégias importantes para aumentar o conhecimento e as práticas dos clínicos no rastreamento da $\mathrm{TB}^{24}$ e no tratamento supervisionado. ${ }^{25}$

Muitas das pesquisas que utilizam o questionário KAP não apresentam os resultados sobre as atitudes devido ao risco substancial de falsamente generalizar as opiniões e sentimentos de um determinado grupo populacional. $\mathrm{O}$ ato de mensurar atitudes ou sentimentos através de pesquisas tem sido criticado por vários motivos: os entrevistados tendem a dar respostas que acreditam serem corretas, aceitas ou apreciadas e os temas delicados são particularmente exigentes, podendo haver influência do contexto na entrevista. ${ }^{26}$ Esta situação ocorreu durante a aplicação do questionário nesta pesquisa, percebia-se certo mal-estar entre os participantes quando confrontados com alguma pergunta delicada, e tendiam a responder algo sobre o que acreditavam ser correto, aceito ou apreciado. Este comportamento foi observado entre os detentos e funcionários do presídio referente às perguntas: "qual o sentimento que você tem sobre as pessoas doentes com TB" e "como uma pessoa que tem TB é considerada pelas outras pessoas". Na primeira questão, o grande número de respostas "solidário e desejo ajudar" levou a crer que esta seria uma afirmação que o participante acreditou ser valorizada no contexto da entrevista, mas que poderia não refletir a sua real atitude. Na segunda pergunta, os detentos responderam que "muitos são rejeitados" e os funcionários do presídio disseram que "muitos ajudam”, mas parte destes também afirmou que "muitos rejeitam". Os participantes poderiam estar desinformados sobre o assunto e considerarem estranha a pergunta, tendo respondido aquilo que julgaram ser adequado no momento da entrevista, porém não expressando a verdade.

Sobre o que sentiriam se adoecessem 
por $\mathrm{TB}$, os detentos referiram tristeza, sentimento que poderia estar associado à condição de confinamento e à própria segurança e sobrevivência, comprometendo a sua relação com os outros detentos ${ }^{4}$. Os funcionários do presídio e da RPS referiram tristeza, surpresa e medo. Novamente, as respostas poderiam não expressar a realidade, mostrando a fragilidade do instrumento na coleta de informações sobre atitudes. $\mathrm{O}$ sentimento de medo é a causa mais comum de estigma na TB. ${ }^{27}$ Para Ascuntar et al., ${ }^{28}$ este sentimento é agregado a um conjunto de atitudes que podem interferir nas relações interpessoais, podendo aumentar os comportamentos de risco, sugerindo a geração do estigma e a discriminação, dificultando o acesso ao tratamento e diminuindo a adesão.

Observou-se que a maior parte dos detentos e funcionários do presídio acreditava em práticas preventivas equivocadas em relação à TB, tais como ter boa higiene e boa alimentação, lavar das mãos, evitar friagem e compartilhamento de pratos e talheres. Na RPS eram esperados melhores resultados sobre o conhecimento das formas de prevenção, porém foi referida a associação do contágio ao compartilhamento de pratos e talheres. Nestes resultados não fica esclarecida a lógica que permeou a tomada de decisão em relação à prevenção, mas indica haver deficiência de conhecimento do manejo clínico e epidemiológico da doença entre os grupos analisados. ${ }^{13}$

Em relação às atitudes, o ato de falar da doença e para quem falaria caso tivesse $\mathrm{TB}$, os detentos referiram a necessidade de comunicar aos seus companheiros de cela ou a qualquer outra pessoa que pudesse ouvi-los sobre a sua condição de doente. Este comportamento pode ser explicado pela fragilidade que a TB representa no contexto prisional. ${ }^{4} \mathrm{O}$ doente pode colocar em risco seus companheiros de cela e, ao se expressar sobre sua doença, estes podem se mobilizar para sua remoção reduzindo a chance de contágio dos outros detentos e colaborar para o rápido acesso ao serviço de saúde prisional. Os funcionários do presídio falariam sobre a doença para um amigo ou para familiares próximos e, na RPS, para médico e parentes. Esta diferença nos resultados reflete uma relação entre o conhecimento sobre a TB e o acesso aos serviços de saúde. ${ }^{18}$

Outra inquietação é que os dados obtidos numa pesquisa KAP são frequentemente utilizados para planejar atividades focadas em mudanças de comportamento em relação a alguma questão da saúde, com base na falsa premissa de que existe uma relação direta entre conhecimento e comportamento. Alguns estudos têm mostrado que o conhecimento é apenas um fator que influencia as práticas e, considerando as mudanças de comportamento, os programas de saúde necessitam relacionar fatores socioeconômicos, ambientais e estruturais às práticas no planejamento das ações de prevenção. $^{18}$

\section{Conclusão}

Neste estudo, a utilização do KAP apresentou vários problemas, dificuldades e limitações. Mostrou-se frágil na interpretação dos dados coletados sobre atitudes, dificultando a compreensão das informações obtidas. Assim, se o objetivo é estudar o comportamento, práticas e atitudes, considerando um determinado contexto, seria indicada a combinação de métodos quali e quantitativos.

Dado que o KAP revelou aspectos importantes sobre conceitos equivocados na tuberculose, tanto entre funcionários da saúde quanto de presídio como de detentos, cabe sugerir sua utilização periódica para auxiliar nas atividades educativas. Considerando a elevada prevalência de TB entre os detentos, aponta-se para a necessidade do envolvimento das Secretarias de Saúde na supervisão de atividades educativas no sistema prisional.

Agradecimentos: Ao Projeto ICOHRTA Aids/TB-Brasil, pelo curso realizado na Johns Hopkins University pelos autores Letícia Marin-León e Sergio Ferreira Júnior. 


\section{Referências}

1. Dara M, Grzemska M, Kimerling ME, Reyes H, Zagorskiy A. Guidelines for control of tuberculosis in prisons. Tuberculosis Coalition for Technical Assistance and International Committee of the Red Cross. 2009. Disponível em: http://pdf.usaid.gov/pdf_docs / PNADP462.pdf. [Acessado em 18 de agosto de 2011]

2. Bick JA. Infection control in jail and prisons. Clin Infect Dis 2007; 45(8): 1047-55.

3. Hanau-Berçot B, Grémy I, Raskine L, Bizet J, Gutierrez MC, Boyer-Mariotte S et al. A one-year prospective study (1994-1995) for a first evaluation of tuberculosis transmission in French prisons. Int J Tuberc Lung Dis 2000; 4(9): 853-9.

4. Diuana V, Lhullier D, Sánchez AR, Amado G, Araújo L, Duarte AM, et al. Saúde em prisões: representações e práticas dos agentes de segurança penitenciária no Rio de Janeiro, Brasil. Cad Saúde Pública 2008; 24: 1887-96.

5. Jittimanee XS, Nateniyon S, Kittikraisak W, Burapat C, Akksilp S, Chumpathat $\mathrm{N}$ et al. Social stigma and knowledge of tuberculosis and HIV among patients with both diseases in Thailand. PLoS One 2009; 23: 4(7): e6360.

6. Baral SC, Karki DK, Newell JN. Causes of stigma and discrimination associated with tuberculosis in Nepal: a qualitative study. BMC Public Health 2007; 7: 211.

7. Pôrto A. Representações sociais da tuberculose: estigma e preconceito. Rev Saúde Pública 2007; 41(1): 43-9.

8. Goffman E. Estigma. Notas sobre a manipulação da identidade deteriorada. $2^{\text {a }}$ ed. Rio de Janeiro: Zahar Editores; 1978.

9. Souza NJ, Bertolozzi MR. A vulnerabilidade à tuberculose em trabalhadores de enfermagem em um hospital universitário. Rev Latino-am Enfermagem 2007; 15(2): 259-66.

10. Alvarez-Gordilho GC, Alvarez-Gordilho FJ, DorantesJiménez JE, Halperin-Frish D. Percepciones y prácticas relacionadas com la tuberculosis y la aderência al tratamiento em Chiapas, México. Salud Pública Méx 2000; 42(6): 520-8.

11. Savicevic AJ, Popovic-Grle S, Milovac S, Ivcevic I, Vukasovic M, Viali V et al. Tuberculosis knowledge among patients in out-patient settings in Split, Croatia. Int J Tuberc Lung Dis 2008; 12(7): 780-5.

12. World Health Organization. Advocacy, communication and social mobilization for TB Control. A guide to developing knowledge, attitude and practice surveys. $\mathrm{WHO} / \mathrm{HTM} / \mathrm{STB} / 2008.46$.

13. Launiala A. How much can a KAP survey tell us about people's knowledge, attitudes and practices? Some observations from medical anthropology research on malaria in pregnancy in Malawi. Anthropology Matters 2009; 11(1): 1-13.
14. Roy A, Abubakar I, Yates S, Chapman A, Lipman M, Monk P et al. Evaluating knowledge gain from TB leaflets for prison and homeless sector staff: the National Knowledge Service TB pilot. Eur J Public Health 2008: 18(6): 600-3

15. Brasil. Ministério da Justiça - Execução Penal. Sistema Prisional. Infopen - Estatística. Disponível em: http://portal.mj.gov.br/data/Pages/ MJD574E9CEITEMIDC37B2AE94C6840068B1624 D28407509CPTBRIE.htm. [Acessado em 20 de agosto de 2011].

16. Abrahão RMCM. Diagnóstico da tuberculose na população carcerária dos Distritos Policiais da zona oeste da cidade de São Paulo [tese de doutorado]. São Paulo: Faculdade de Saúde Pública da USP; 2003.

17. Brasil. Ministério da Saúde. Plano Nacional de Saúde no Sistema Penitenciário. Disponível em: http://bvsms. saude.gov.br/bvs/publicacoes/plano_nacional_saude_ sistema_penitenciario_2ed.pdf. [Acessado em $20 \mathrm{de}$ agosto de 2011]

18. Launiala A, Honkasalo ML. Ethnographic study of factors influencing compliance to intermittent preventive treatment of malaria during pregnancy among Yao women in rural Malawi. Trans $R$ Soc Trop Med Hyg 2007; 101(10): 980-9.

19. Abebe DS, Biffa D, Bjune G, Ameni G, Abebe F. Assessment of knowledge and practice about tuberculosis among eastern Ethiopian prisoners. Int J Tuberc Lung Dis 2011; 15(2): 228-33.

20. Waisbord S. Participatory communication for tuberculosis control in prisons in Bolivia, Ecuador, and Paraguay. Rev Panam Salud Publica 2010; 27(3): 168-74.

21. Boaretto MC, Guimarães MTC, Natal S, Castelo Branco AC, Mondarto P, Fernandes MJ et al. The knowledge of the Brazilian population on tuberculosis. Int J Tuberc Lung Dis 2010; 14(11): S196.

22. Maciel ELN, Vieira RCA, Milani EC, Brasil M, Fregona G, Dietze R. O agente comunitário de saúde no controle da tuberculose: conhecimentos e percepções. Cad Saude Publica 2008; 24(6): 1377-86.

23. Brasil. Ministério da Saúde. Tuberculose - Guia de vigilância epidemiológica, 2005. Disponível em http:// www.prosaude.org/publicacoes/guia/Guia_Vig_Epid_ novo2.pdf. [Acessado em 24 de abril de 2011].

24. Naugthon MP, Posey DL, Willacy EA, Comans TW. Tuberculosis training on physicians who perform immigration medical examination. Int J Tuberc Lung Dis 2010; 14(11): S154.

25. Rao N, Arain I. Knowledge regarding tuberculosis among TN course participants in Karachi. Int J Tuberc Lung Dis 2010; 14(11): S160. 
26. Hausmann-Muela S, Ribera JM, Nyamongo I. Health seeking behavior and the health system response. DCPP Working Paper 2003. Disponível em: http://www.dcp2. org/main/Home.html. [Acessado em 24 de abril de 2010].

27. Courtwright A, Turner AN. Tuberculosis and stigmatization: pathways and interventions. Public Health Rep 2010; 125(4): 34-42.
28. Ascuntar JM, Gaviria MB, Uribe L, Ochoa J. Fear, infection and compassion: social representations of tuberculosis in Medellin, Colombia, 2007. Int J Tuberc Lung Dis 2010; 14(10): 1323-29.

Recebido em: 29/09/11

Versão final apresentada em: 19/12/11

Aprovado em: 08/02/12 